\title{
REAL RELATIVE COHOMOLOGY OF FINITE-CODIMENSION GERMS
}

\author{
BY CLAUDE ANDRÉ ROCHE
}

1. Relative and vanishing cohomology. Let $P$ be a germ at $0 \in \mathbf{R}^{n}$ of a $C^{\infty}$ function of finite codimension (see [7]). Let $\varepsilon_{n}$ be the ring of germs at 0 of smooth functions: $\mathbf{R}^{n} \rightarrow \mathbf{R}, m_{n}^{\infty}$ the ideal of flat germs of $\mathcal{E}_{n}$ (i.e. with null Taylor expansion) and $\xi_{n}$ the ring of real formal series in $n$ indeterminates $X_{1}, \ldots, X_{n}$. Set $\Lambda^{*}$ (respectively $\Lambda_{\infty}^{*}, \hat{\Lambda}^{*}$ ) to be the de Rham complex of germs of smooth forms (resp. of forms of $m_{n}^{\infty} \Lambda$, of "forms" in the module over $\mathcal{F}_{n}$ spanned by $\left.d X_{1}, \ldots, d X_{n}\right)$. We construct a relative de Rham complex $\left(\Lambda_{\text {rel }}^{*}, d_{\text {rel }}\right)$ (resp. $\left.\left(\Lambda_{\infty \text { rel }}^{*}, d_{\text {rel }}\right),\left(\hat{\Lambda}_{\text {rel }}^{\cdot}, d_{\text {rel }}\right)\right)$ using Leibniz rule, $\Lambda_{\text {rel }}^{\prime}=\Lambda^{*} / d P \wedge \Lambda^{*}$ (resp. $\Lambda_{\infty \text { rel }}^{*}=\Lambda_{\infty}^{\prime} / d P \wedge \Lambda_{\infty}^{*}, \hat{\Lambda}_{\text {rel }}^{*}=\hat{\Lambda}^{\cdot} / d j^{\infty} P \wedge \hat{\Lambda}^{*}, j^{\infty}$ the jet epimorphism, $\mathcal{E}_{n} \rightarrow \mathcal{F}_{n}$ ) this module is endowed with a natural structure of $\mathcal{E}_{1}$ (resp. $m_{1}^{\infty}$, $f_{1}$ )-module through $P$. By the chain rule $d_{\text {rel }}$ is linear. Define $H_{\text {rel }}$ (resp. $H_{\infty \text { rel }}^{\cdot}, \hat{H}_{\text {rel }}^{\cdot}$ ) as the cohomology module of the relative de Rham complex of smooth (resp. flat, formal) forms.

Relative cohomology appears in the study of hypersurface singularities. In the complex case, E. Brieskorn [2] proved that holomorphic relative cohomology is determined by the formal one (Bloom's theorem). In fact this cohomology is completely determined by the vanishing cohomology sheaf in the case of an isolated singularity (Brieskorn-Sebastiani).

We can define the real vanishing cohomology of $P$ as the stalk at 0 of the sheaf $R^{\prime} P_{*}(\underline{\mathbf{R}})$, where $\underline{\mathbf{R}}$ is the constant sheaf on a small ball $B$ at the origin of $\mathbf{R}^{n}$. By the fibration theorem, $P$ if restricted to $B \cap P^{-1}((-\eta, \eta)-\{0\})$ with $\eta$ small, is a fibre projection and $R \cdot P_{*}(\underline{\mathbf{R}})$ a real local system. Hence $R^{i} P_{*}(\underline{\mathbf{R}})$ is trivial over $(-\eta, 0)$ and $(0, \eta)$, the stalk being isomorphic to $\mathbf{R}^{a_{-i}}$ and $\mathbf{R}^{a_{i}}$ respectively. Let $b_{i}$ be $a_{-i}+a_{i}$ then $b_{i}$ is the dimension of the $i$ th vanishing cohomology space.

THEOREM 1. The $m_{1}^{\infty}$-module $H_{\infty \text { rel }}^{k}$ is free of rank $b_{k}$.

THEOREM 2. (a) The sequence $0 \rightarrow \Lambda_{\infty \mathrm{rel}} \rightarrow \Lambda_{\text {rel }} \rightarrow \hat{\Lambda}_{\text {rel }} \rightarrow 0$ is exact over $0 \rightarrow m_{n}^{\infty} \rightarrow \varepsilon_{n} \rightarrow \mathcal{F}_{n} \rightarrow 0$.

(b) The long exact homology sequence associated to it has null connecting morphism.

The first part is easy for finite-codimension germs and false for infinitecodimension ones. The second uses the result of Bloom-Brieskorn-Sebastiani except in degree 0 . In this case a result of Moussu on Left equivalence of germs is used.

Received by the editors May 26, 1982.

1980 Mathematics Subject Classification. Primary 58C27, 32B30; Secondary 32C05. 
COROLLARY. The relative cohomology of smooth forms $H_{\mathrm{rel}}^{\cdot}$ is an extension of $\hat{H}_{\text {rel }}^{\cdot}$ by $H_{\text {c rel }}$.

2. Clemens structure over a divisor with normal crossings. Let $p$ be a polynomial germ right equivalent to $P$. We shall use a resolution of the singularity of $(p)$ (Hironaka's theorem), $\theta: \tilde{U} \rightarrow U$ for $U$ a suitable neighborhood of $0 \in \mathbf{R}^{n}$. Let $D$ be the total transform of $(p)$ (determined by $q=p \circ \theta$ ) and $E$ the exceptional divisor of $\theta$. D has a decomposition $E \cup \tilde{X}_{0}$ where $\tilde{X}_{0}-\left(E \cap \tilde{X}_{0}\right)$ is isomorphic to $\{p=0\}-\{0\}$ and $D$ has normal crossings.

Consider now any analytic divisor with normal crossings $D$ in a real analytic manifold $\tilde{U}$.

DEFinition. A Clemens structure over $D$ in $\tilde{U}$ is a functor assigning to each natural stratum $X$ of $D$ a germ of tubular neighborhood, $C(X)$ together with a $C^{\infty}$ vector field $\xi_{x}$ on it. Local models are $\xi_{x}=\sum r_{i} / y_{i} \partial / \partial y_{i}$ if $D$ is (locally) defined by $y_{1}^{r_{1}} \ldots y_{c}^{r_{c}}$ ( $c$ is then the codimension of $X$ in $\tilde{U}$ ). The structural group of $C(X)$ is the group of all permutations up to sign of $y_{1}, \ldots, y_{c}$. It is isomorphic to a semidirect product of $\mathbf{Z}_{2}^{c}$ by the permutation group of $c$ elements. To each incidence relation of natural strata $Y \leq X$ we assign an open immersion $C(Y) \rightarrow C(X)$ mapping $\xi_{Y}$ onto $\xi_{X}$.

This definition differs from that of Arnold [1] in that the role played by the retraction deduced from the field $\xi$ (obtained by patching the $\xi_{x}$ ) is emphasized.

THEOREM 3. There exist a Clemens structure over $D$ in $\tilde{U}$.

The proof is by induction on the dimension of the strata as in Clemens paper [3] or in [8].

A definition of maps with moderate growth in $D$ obtained by generalizing those in [5] allows us to handle flat forms. We thus obtain a one-to-one correspondence between forms flat on $D$ and forms flat on $q^{-1}(\lambda) \times\{0\}$.

PROPOSITION. Let $\varphi(x, t)$ be the flow of $\xi$ in a neighborhood of $D$ in $\tilde{U}$, $t(x, \eta)$ the function defined by the implicit relation $|q(\varphi(x, t(x, \eta)))|=\eta$. Then there exists $\lambda>0$ so that the map

$$
\Phi: \tilde{U}-D \rightarrow q^{-1}(\lambda) \times\{(-\lambda, \lambda)-\{0\}\}: \quad x \mapsto(\varphi(x, t(x, \lambda)), q(x))
$$

(resp. its inverse) has moderate growth in $D$ (resp. in $\left.q^{-1}(\lambda) \times\{0\}\right)$.

Next we prove the nullity of transversally formal relative cohomology (by using a Clemens structure over $E \cap \tilde{X}_{0}$ in $\tilde{X}_{0}$ ). This means that we have

LEMMA. Each class in $H_{\infty \text { rel }}^{k}$ contains a form of $m_{1}^{\infty} \Lambda^{k}$.

Then the proposition above allows the proof of Theorem 1 to be reduced to a problem with forms of $\Lambda^{\cdot} q^{-1}(\lambda) \times\{[-1,1]-\{0\}\}$ flat in $q^{-1}(\lambda) \times\{0\}$. The use of a theory of de Rham-Hodge for the manifold with boundary $q^{-1}(\lambda)$ gives a choice of forms over $q^{-1}(\lambda) \times([-1,1]-\{0\})$. These forms give a basis for the vanishing cohomology and give also the isomorphism of the relative cohomology with $\left(m_{1}^{\infty}\right)^{b_{k}}$. 
3. Local simultaneous reduction of a function and a volume form. We generalise the result of Colin de Verdière and Vey [4] about the reduction of a Morse germ to a quadratic form by diffeomorphisms preserving a fixed volume form. This we do by giving the normal forms of a volume form under the action of the group of all the diffeomorphisms stabilizing a given germ of function of finite codimension. This is a consequence of the structure of $H_{\infty \text { rel }}^{n-1}($ compare $[4,6]$ ). Let $P$ be a codimension $\mu$ germ and $I(P)$ the group mentioned above.

THEOREM 4. (I) Let the germ of $\{P=0\}$ be different from $\{0\}$. There exist $\mu$ germs of $n$-forms $w_{i}$ so that for each volume form $w$ there exist $\varphi \in I(P)$ and $\psi_{i} \in \mathcal{E}_{1}$ such that $\varphi^{*} w=\sum \psi_{i}(P) w_{i}$.

(II) If the germ of $\{P=0\}$ is $\{0\}$ then there exist $\mu$ germs of $n$-forms $w_{i}$ and a germ of $n-1$-form $w_{0}$ differentiable except at 0 , moderated at 0 so that for each volume form $w$ there exist $\varphi \in I(P)$ and $\psi_{i} \in \mathcal{E}_{1}(i>0), \psi_{0} \in m_{1}^{\infty}$ such that $\varphi^{*} w=\sum \psi_{i}(P) w_{i}+d\left(\psi_{0}(P) w_{0}\right)$.

REMARK. The form $w_{0}$ is moderate in the sense above so that the forms in $m_{1}^{\infty} w_{0}$ are smooth at zero.

\section{REFERENCES}

1. V. I. Arnold, Index of singular points of a vector field, the Petrovskx-Oletnik inequalities and mixed Hodge structures, Funkcional Anal. i Prilozen 12 (1978), 1-14; English transl. in Functional Anal. Appl. 12 (1978),

2. E. Brieskorn, Die Monodromie der isolierten Singularitäten von Hyperflächen, Manuscripta Math. 2 (1970), 103-161.

3. C. H. Clemens, Picard-Lefschetz theorem for families of nonsingular algebraic varieties acquiring ordinary singularities, Trans. Amer. Math. Soc. 136 (1969), 93-108.

4. Y. Colin de Verdiere and J. Vey, Le lemme de Morse isochore, Topology 18 (1979), 283-293.

5. P. Deligne, Equations différentielles d points singuliers reguliers, Lecture Notes in Math., vol. 136, Springer-Verlag, Berlin and New York, 1970, pp.

6. J. P. Françoise, Modele local simultané d'une fonction et d'une forme de volume, Astérisque S.M.F. 59-60 (1978), 119-130.

7. Jean Martinet, Singularities of smooth functions and maps, London Math. Soc. Lecture Notes 58, Cambridge Univ. Press, 1981.

8. V. A. Vasilev, Asymptotic behavior of exponential integrals in the complex domain, Functional Anal. Appl. 13 (1979), 239-247.

LABORATOIRE DE MAThEMATIQUES PURES-INSTITUT FOURIER DÉPENDANT DE L'UNIVERSITE SCIENTIFIQUE ET MÉDICALE DE GRENOBLE ASSOCIE AU C.N.R.S., B.P. 116, 38402 St. MARTIN D'HERES, FranCE 